\title{
Estimativa da Área Foliar de Cinco Espécies do Gênero amaranthus usando Dimensões Lineares do Limbo FoliaR ${ }^{1}$
}

\author{
Leaf Area Estimation of Five Amaranthus Species Using Leaf Blade Linear Dimensions
}

CARVALHO, S.J.P. ${ }^{2}$ e CHRISTOFFOLETI, P.J. ${ }^{3}$

\begin{abstract}
RESUMO - Este trabalho foi realizado com o objetivo de obter equações que estimem a área foliar de espécies de plantas daninhas do gênero Amaranthus utilizando as dimensões lineares do limbo foliar, bem como comparar as equações obtidas para plantas que cresceram em casa de vegetação e em campo. Para isso, plantas de A. deflexus, A. hybridus, A. retroflexus, A. spinosus e $A$. viridis foram cultivadas em casa de vegetação ou coletadas em infestações naturais de áreas agrícolas e não-agrícolas do município de Piracicaba-SP. Para cada condição foram analisados 100 limbos foliares, com relação a comprimento ao longo da nervura principal $(C)$, largura máxima $(L)$ e área foliar real $(A r)$. Os conjuntos de dados foram ajustados à equação linear que passa pela origem $A r=a .(C . L)$ e, para todas as variáveis, comparados por meio de intervalos de confiança, a $5 \%$ de significância. Concluiu-se que: há espécies de plantas daninhas do gênero Amaranthus com tamanhos diferenciais de folhas, sendo A. deflexus aquela que, em média, possui as menores dimensões; a equação linear passando pela origem $(A r=a .(C . L))$ foi adequada para ajustar a relação entre as medidas lineares do limbo e a área foliar real; e plantas que cresceram em casa de vegetação e em campo podem apresentar diferenças quanto ao tamanho de folhas ou quanto ao parâmetro $a$ da equação de ajuste da reta.
\end{abstract}

Palavras-chave: caruru, folhas, crescimento, ajuste linear.

ABSTRACT - This work aimed to obtain equations to estimate the leaf area of weed species of the Amaranthus genus, using leaf blade linear dimensions, as well as to compare the equations set up for plants grown under greenhouse and field conditions. Thus, plants of A. deflexus, A. hybridus, $\boldsymbol{A}$. retroflexus, $\boldsymbol{A}$. spinosus and $\boldsymbol{A}$. viridis were grown in the greenhouse or collected in natural infestations of agricultural or non-agricultural areas of Piracicaba-SP, Brazil. For each condition, maximum length $(C)$ and width $(L)$ dimensions, as well as real leaf area (Ar) per leaf were measured in 100 leaf blades. The data groups were adjusted to linear equation crossing the origin $\mathrm{Ar}=a .(C . L)$ and compared through confidence intervals of 5\% significance for all variables. It was concluded that: weed species of the Amaranthus genus display differential leaf sizes, with $\boldsymbol{A}$. deflexus presenting the smallest dimensions, on average; the linear equation crossing the origin $(A r=a .(C . L))$ was adequate to adjust the relation between linear blade dimensions and real leaf area; plants grown under greenhouse and field conditions may present differences in leaf size or in the $\boldsymbol{a}$ parameter of the adjusted equation.

Keywords: pigweed, leaves, growth, linear adjustment.

\section{INTRODUÇÃO}

As plantas classificadas no gênero Amaranthus (carurus) são espécies vegetais que têm por centro de origem a região da América tropical, onde ocorrem em intensas popula- ções. Nesse gênero de plantas são encontradas algumas espécies de interesse agronômico que apresentam elevado valor nutricional, como A. caudatus, A. cruentus e A. hypochondriacus. Essas espécies podem ser utilizadas na alimentação humana e animal, bem como

Recebido para publicação em 13.9.2006 e na forma revisada em 17.4.2007.

2 Eng ${ }^{\mathrm{e}}$-Agr ${ }^{\circ}$, Mestre em Fitotecnia pela ESALQ/USP, Bolsista FAPESP < sjpcarvalho@yahoo.com.br>; ${ }^{3}$ Professor Associado do Dep.de Produção Vegetal, ESALQ/USP. <pjchrist@esalq.usp.br> Caixa Postal 09, 13418-900, Piracicaba-SP. 
para produção de massa vegetal, fatores esses que estimularam o desen volvimento de cultivares comerciais (Kissmann \& Groth, 1999; Spehar, 2003; Spehar et al., 2003).

Contudo, embora apresente aspectos positivos, a característica mais reconhecida do gênero Amaranthus está relacionada com as espécies que apresentam potencial competitivo com as mais diversas culturas. Mundialmente, existem cerca de 20 espécies classificadas como plantas daninhas, sendo assim denominadas em mais de 50 países. No Brasil, os carurus são conhecidos em praticamente todas as regiões, com algumas diferenças de espécies entre as localidades, em que cerca de 10 espécies têm sido destacadas como infestantes de maior importância (Kissmann \& Groth, 1999).

O hábito de crescimento agressivo e a prolifica produção de sementes oferecem às plantas de Amaranthus boa competitividade com as culturas por luz, água e nutrientes (Murphy et al., 1996; Guo \& Al-Khatib, 2003). Dessa forma, reduzem o rendimento, a qualidade e também a eficiência de colheita das plantas cultivadas (Klingaman \& Oliver, 1994; Knezevic et al., 1997). Por fim, espécies do gênero Amaranthus têm apresentado efeitos alelopáticos que reduzem o vigor das plântulas de várias culturas e de outras plantas daninhas (Menges, 1988; Marques, 1992).

Em geral, quando se estudam programas para o manejo integrado de plantas daninhas em agroecossistemas, os conhecimentos sobre a biologia das espécies envolvidas têm grande importância (Bianco et al., 1995). Contudo, uma das maiores limitações que existem para a implantação desses programas de manejo é a própria carência de conhecimentos sobre a biologia das espécies (Fernández, 1982).

Nesse sentido, estudos sobre o crescimento e o desenvolvimento das plantas daninhas fornecem informações acerca dos diferentes estádios fenológicos e padrões de crescimento, que tornam possível a análise do comportamento dessas plantas perante os fatores ecológicos, bem como de sua ação sobre o ambiente, principalmente quanto à sua interferência em outras plantas (Lucchesi,
1984). Para isso, Radosevich et al. (1997) afirmam que a produção de massa seca total e o acúmulo de área foliar são reconhecidos como processos básicos no crescimento vegetal.

Dos parâmetros de crescimento vegetal, a área foliar é um dos mais importantes, sendo também uma das características mais dificeis de ser mensurada, porque usualmente exige equipamentos caros ou técnicas destrutivas, que impedem a comparação de efeitos em um mesmo indivíduo (Bianco et al., 1995, 2003). Uma técnica não-destrutiva utilizada com bastante eficiência é a obtenção de equações que correlacionem as medidas dimensionais das folhas com sua área foliar real (Bianco et al., 2004).

Assim, este trabalho teve por objetivo obter equações que estimem a área foliar de espécies de plantas daninhas do gênero Amaranthus utilizando as dimensões lineares do limbo foliar, bem como comparar as equações obtidas para plantas que cresceram em casa de vegetação e em campo.

\section{MATERIAL E MÉTODOS}

Os experimentos foram conduzidos em casa de vegetação e em área experimental do Departamento de Produção Vegetal da Escola Superior de Agricultura "Luiz de Queiroz" ESALQ/USP e em áreas agrícolas e nãoagrícolas do município de Piracicaba-SP (22 42'30'S, 47³8'00'W), entre os meses de setembro e dezembro de 2005. As espécies de plantas daninhas estudadas foram: A. deflexus (caruru-rasteiro), A. hybridus (caruru-roxo), A. retroflexus (caruru-gigante), A. spinosus (caruru-de-espinho) e A. viridis (caruru-demancha).

\section{Crescimento em casa de vegetação}

Para a condição de casa de vegetação, os diásporos das espécies $A$. hybridus, $A$. spinosus e $A$. viridis foram adquiridos comercialmente, enquanto os diásporos de $A$. deflexus e A. retroflexus foram coletados em infestações presentes em área da ESALQ/USP - Piracicaba-SP (A. deflexus) e em áreas agrícolas dos municípios de Miguelópolis e Guará-SP (A. retroflexus). As espécies foram taxonomicamente identificadas e seus propágulos foram 
acondicionados em sacos de papel, em local seco, à temperatura ambiente, até o início da instalação dos experimentos. No experimento, foram utilizadas sementes de A. hybridus, A. retroflexuse $A$. viridise frutos de $A$. deflexus e A. spinosus, respeitando-se a principal forma de dispersão das espécies.

Em 1.9.2005, as sementes ou frutos das cinco espécies de carurus foram colocados para germinar em caixas plásticas, com capacidade para $2 \mathrm{~L}$, preenchidas com substrato comercial (casca de pínus + turfa + vermiculita). No estádio de folhas cotiledonares plenamente expandidas, ou seja, estádio 10 (Hess et al., 1997), as espécies foram transplantadas para vasos, onde permaneceram até o final do experimento. Assim, o transplantede A. hybridus, A. retroflexus e $A$. viridis foi realizado aos sete dias após a semeadura (DAS), enquanto o de A. spinosus e A. deflexus se deu aos 10 DAS. Foram transplantadas duas plantas por vaso, porém, aos 20 DAS, efetuou-se o desbaste da planta menos desenvolvida.

Para cada espécie, as parcelas experimentais constaram de 30 vasos plásticos com capacidade para $2,8 \mathrm{~L}$, preenchidos com mistura de substrato comercial e vermiculita, na proporção de 2:1, respectivamente. Três dias antes do início dos transplantes, os vasos foram adubados com $4 \mathrm{~g}$ de fertilizante comercial completo, que forneceu as seguintes doses de nutrientes (mg vaso-1): $\mathrm{N}(600) ; \mathrm{P}_{2} \mathrm{O}_{5}(600) ; \mathrm{K}_{2} \mathrm{O}$ (800); Ca (44); S (160); Mg (16); Zn (2); B (2); Fe (4); e Mn $(1,2)$. Aos 35 DAS, realizou-se adubação complementar com sulfato de amônio nas doses de 315 e $360 \mathrm{mg}$ parcela $^{-1}$ de N e S, respectivamente. A adubação utilizada contribuiu para o desenvolvimento das plantas, sem a manifestação de deficiência nutricional. Os vasos foram irrigados sempre que se julgou necessário, sem a ocorrência de deficiência hídrica.

\section{Crescimento em campo}

Foram utilizadas infestações naturais das espécies $A$. deflexus, $A$. spinosus e $A$. viridis com ocorrência nas áreas experimentais do Departamento de Produção Vegetal da ESALQ/ USP ou nas áreas agrícolas e não-agrícolas do município de Piracicaba. Não foram encontradas infestações representativas das espécies
A. hybridus e A. retroflexus, de modo que não foram obtidas equações para as folhas destas espécies quando se desenvolveram em campo.

\section{Coletas de folhas e análises}

Foram coletados 100 limbos foliares perfeitos (sem deformações) de cada uma das espécies de plantas daninhas, em cada uma das condições de crescimento. Em casa de vegetação, das 30 parcelas disponiveis, foram escolhidas seis parcelas perfeitamente representativas, visando reduzir a variabilidade dos dados, cujas plantas se encontravam sadias, sem o ataque de pragas ou patógenos que pudessem danificar o formato das folhas. Aos 60 DAS, foram coletadas folhas do terço superior, intermediário e basal das plantas, em igual proporção. Identificou-se estádio fenológico de pleno florescimento, estádio 69 (Hess et al., 1997), ou superior, para todas as espécies.

Em campo, foram coletadas folhas de plantas que cresceram em diferentes condições ambientais, em que também foram retiradas folhas do terço superior, intermediário e basal. Também nesse caso, os indivíduos escolhidos para coleta apresentavam estádio fenológico de pleno florescimento ou mais avançado.

As coletas foram realizadas em fluxos de 100 limbos ou menos, a fim de evitar a deterioração do material. Logo após a coleta, o material foi levado ao laboratório, onde se realizou a medição do comprimento ao longo da nervura central $(C)$ e largura máxima $(L)$ de cada folha. Em seguida, a area foliar real $(A r)$ de cada folha foi obtida com auxílio do medidor de área modelo LICOR LI-3100 (LI-COR, inc., Lincoln, Nebraska, EUA).

Os dados foram submetidos à análise de regressão linear, adotando-se a seguinte equação:

$$
A r=a .(C . L)
$$

em que $A r$ é a área foliar real e $a$, um parâmetro da reta que corresponde ao percentual de equivalência entre a área foliar real e o produto do comprimento $(C)$ pela largura $(L)$. Toda a metodologia de coleta e análise foi baseada em trabalhos tradicionalmente descritos na literatura, como em Peressin et al. (1984) e Bianco et al. (1995, 2004). 
A análise das retas foi feita por meio da comparação dos intervalos de confiança obtidos para os parâmetros $a$, a $5 \%$ de significância. Para cada espécie, na ocorrência da sobreposição do intervalo de confiança de duas retas (campo e casa de vegetação), julgou-se a igualdade das equações e obteve-se uma terceira reta com a totalidade dos pontos amostrais (casa de vegetação + campo). Quando não ocorreu a sobreposição dos intervalos de confiança, julgou-se a existência de duas retas diferentes. O mesmo procedimento foi adotado para os dados de comprimento, largura de folhas e área foliar.

\section{RESULTADOS E DISCUSSÃO}

As folhas das plantas daninhas do gênero Amaranthus, em geral, são do tipo simples e alternas, com limbo ovalado, rômbico ou rômbico-lanceolado; as superficies são glabras ou com escassa pilosidade e com ocorrência comum de ápice obtuso. Apresentam pecíolo longo, que, por vezes, pode exceder o comprimento do limbo, canaliculado no lado superior. As nervuras são marcadas na face ventral, especialmente nas folhas novas, e proeminentes na dorsal. A coloração dos limbos varia entre verde, verde-acinzentada, avermelhada, vermelho-púrpura (A. hybridus) ou com a ocorrência de manchas (A. viridis) (Kissmann \& Groth, 1999).

Com relação a $A$. deflexus, constatou-se que as folhas provenientes das plantas que cresceram em casa de vegetação foram significativamente maiores que aquelas coletadas em plantas que cresceram em campo. Isso se manifestou sobre o comprimento, a largura e, por conseqüência, a área foliar (Tabela 1). Embora tenha apresentado folhas de tamanhos diferentes entre as condições de casa de vegetação e campo, o parâmetro de ajuste da reta não foi diferente nas duas condições, de modo que todos os pontos foram ajustados em conjunto e obteve-se a equação $A r=0,7579$. (C.L) (Tabela 2), representada graficamente pela Figura 1. Dentre as espécies, em média, constatou-se que $A$. deflexus possui folhas menores que $A$. hybridus, $A$. retroflexus, $A$. viridis e $A$. spinosus (casa de vegetação), principalmente quanto ao comprimento (Tabela 1).
As espécies $A$. hybridus, A. retroflexus e A. viridisforam aquelas que, em média, apresentaram os maiores valores de comprimento e área foliar, sem diferenças interespecíficas (Tabela 1). As retas obtidas para $A$. hybridus e A. retroflexus foram $A r=0,7056 .(C . L) \mathrm{e}$ $A r=0,7005 .(C . L)$, também sem diferenças interespecíficas entre si (Tabela 2), e estão representadas graficamente nas Figuras 2 e 3.

Dentre as espécies de Amaranthus, somente foi encontrado trabalho que estudou a espécie A. retroflexus. A equação obtida para a população que cresceu em casa de vegetação diferenciou-se sobremaneira do trabalho de Bianco et al. (1995), que obtiveram a equação $A r=0,5651$.(C.L) para plantas com crescimento em campo. Com base nos trabalhos, não se pode isolar os fatores que causaram a diferença nas equações, porém sugere-se que as diferenças sejam devidas à metodologia experimental (equipamento medidor de área foliar) e às condições diferenciais de ambiente em campo e casa de vegetação.

Os resultados obtidos para $A$. spinosus estão em concordância com o que foi observado para A. deflexus. Também nesse caso, as folhas coletadas de plantas que cresceram em casa de vegetação apresentaram maiores dimensões quanto a comprimento ao longo da nervura principal, largura máxima e área foliar (Tabela 1). Contudo, novamente, não foram observadas diferenças quanto ao parâmetro de ajuste da reta, o que indica que as folhas provenientes de ambas as condições de crescimento apresentam a mesma proporção C.L/ $A r$. Nesse caso, uma única equação foi satisfatória para as duas condições, sendo $A r=0,7150 .(C . L)$ o resultado do ajuste matemático (Tabela 2 ), que está representado graficamente na Figura 4.

Para A. viridis, em oposição ao que foi observado em $A$. deflexus e $A$. spinosus, foram constatadas diferenças entre as folhas oriundas de plantas que cresceram em casa de vegetação e campo somente para o parâmetro de ajuste da reta, ou seja, não foram observadas diferenças quanto a comprimento, largura ou área foliar (Tabela 1); todavia, ocorreram alterações na proporção C.L/Ar, em que as plantas que cresceram em campo apresentaram maior parâmetro de ajuste, e a equação obtida foi $A r=0,7110 .(C . L)$, representada pela Figura 5. 
Tabela 1 - Média e intervalo de confiança (IC, 5\%) para comprimento ao longo da nervura central, largura máxima e área foliar real de folhas de plantas daninhas do gênero Amaranthus, coletadas em plantas mantidas sob duas condições de crescimento. Piracicaba, 2005

\begin{tabular}{|c|c|c|c|c|c|c|c|}
\hline \multirow[b]{2}{*}{ Espécie } & \multirow[b]{2}{*}{ Condi ção } & \multicolumn{2}{|c|}{ Comprimento } & \multicolumn{2}{|c|}{ Largura } & \multicolumn{2}{|c|}{ Área Foliar Real } \\
\hline & & Média & $\begin{array}{c} \pm \mathrm{IC} \\
(5 \%)\end{array}$ & Média & $\begin{array}{c} \pm \mathrm{IC} \\
(5 \%)\end{array}$ & Média & $\pm \mathrm{IC}(5 \%)$ \\
\hline \multirow{2}{*}{ A. deflexus } & Casa de vegetação & 6,326 & 0,378 & 5,148 & 0,329 & 27,037 & 3,190 \\
\hline & Campo & 4,956 & 0,244 & 3,800 & 0,200 & 15,162 & 1,543 \\
\hline A. hybridus & Casa de vegetação & 8,214 & 0,803 & 5,164 & 0,520 & 37,734 & 8,296 \\
\hline A. retroflexus & Casa de vegetação & 9,547 & 0,700 & 5,667 & 0,412 & 43,000 & 6,845 \\
\hline \multirow{2}{*}{ A. spinosus } & Casa de vegetação & 8,185 & 0,475 & 5,212 & 0,307 & 33,200 & 3,941 \\
\hline & Campo & 5,806 & 0,218 & 3,494 & 0,150 & 15,000 & 1,123 \\
\hline \multirow{2}{*}{ A. viridis } & Casa de vegetação & 8,900 & 0,764 & 4,908 & 0,431 & 35,679 & 7,099 \\
\hline & Campo & 8,668 & 0,485 & 5,395 & 0,311 & 35,921 & 3,872 \\
\hline
\end{tabular}

Tabela 2 - Parâmetro $a$ da equação de regressão ${ }^{1^{\prime}}$, coeficiente de determinação $\left(\mathrm{R}^{2}\right)$, valor de $\mathrm{F}$ e intervalo de confiança (IC) a $5 \%$ de significância, para estimativa da área foliar de plantas daninhas do gênero Amaranthus a partir das dimensões lineares do limbo foliar. Piracicaba, 2005

\begin{tabular}{|l|c|c|c|c|c|c|}
\hline \multirow{2}{*}{ Espécie } & \multirow{2}{*}{ Condição } & $a$ & \multirow{2}{*}{$\mathrm{R}^{2}$} & \multirow{2}{*}{$\mathrm{F}$} & \multicolumn{2}{|c|}{$\mathrm{IC}(5 \%)$} \\
\cline { 3 - 7 } & & & & \multicolumn{2}{|c|}{ Mín. } & Máx. \\
\hline \multirow{3}{*}{ A. deflexus } & Casa de vegetação & $0,7580^{*}$ & 0,9928 & $13661,53^{* *}$ & 0,7515 & 0,7646 \\
\cline { 2 - 7 } & Campo & $0,7577^{*}$ & 0,9913 & $11324,24^{* *}$ & 0,7513 & 0,7641 \\
\cline { 2 - 7 } & Total & $0,7579^{*}$ & 0,9940 & $32912,21^{* *}$ & 0,7534 & 0,7625 \\
\hline A. hybridus & Casa de vegetação & $0,7056^{*}$ & 0,9978 & $46419,08^{* *}$ & 0,6991 & 0,7121 \\
\hline A. retroflexus & Casa de vegetação & $0,7005^{*}$ & 0,9989 & $96976,87^{* *}$ & 0,6961 & 0,7050 \\
\hline \multirow{3}{*}{ A. spinosus } & Casa de vegetação & $0,7146^{*}$ & 0,9624 & $2557,68^{* *}$ & 0,7003 & 0,7289 \\
\cline { 2 - 7 } & Campo & $0,7172^{*}$ & 0,9808 & $5114,52^{* *}$ & 0,7100 & 0,7244 \\
\cline { 2 - 7 } & Total & $0,7150^{*}$ & 0,9737 & $7443,72^{* *}$ & 0,7055 & 0,7245 \\
\hline \multirow{3}{*}{ A. viridis } & Casa de vegetação & $0,6741^{*}$ & 0,9973 & $36381,46^{* *}$ & 0,6691 & 0,6790 \\
\cline { 2 - 7 } & Campo & $0,7110^{*}$ & 0,9900 & $9475,01^{* *}$ & 0,7091 & 0,7178 \\
\cline { 2 - 7 } & Total & $0,6877^{*}$ & 0,9939 & $32842,30^{* *}$ & 0,6830 & 0,6925 \\
\hline
\end{tabular}

${ }^{1 /} A r=a .(C . L) ; *$ significativo pelo teste t a $5 \%$ de probabilidade; ** significativo pelo teste $\mathrm{F}$ a $5 \%$ de probabilidade.

Nesse caso, o mais adequado é considerar uma equação para cada circunstância, de modo que a equação obtida para a condição de casa de vegetação foi $A r=0,6741$.(C.L) (Tabela 2), representada graficamente pela Figura 6. Também nesse caso, não se pode determinar quais fatores ambientais-ecológicos influenciaram as diferenças de tamanho de folhas identificadas para as espécies $A$. deflexus e $A$. spinosus ou o ajuste da reta para $A$. viridis. Contudo, considera-se que o nivel nutricional oferecido às plantas que cresceram em casa de vegetação pode ter influenciado o desenvolvimento de plantas mais vigorosas e, portanto, com maiores dimensões de folhas, as quais não alteraram a proporção de suas dimensões em relação à área foliar real. Outro fator que também pode ter influenciado as dimensões foliares são as condições de intensidade lu minosa diferencial de casa de vegetação e campo ou o nivel de competição (sombreamento) a que as plantas estiveram submetidas. 


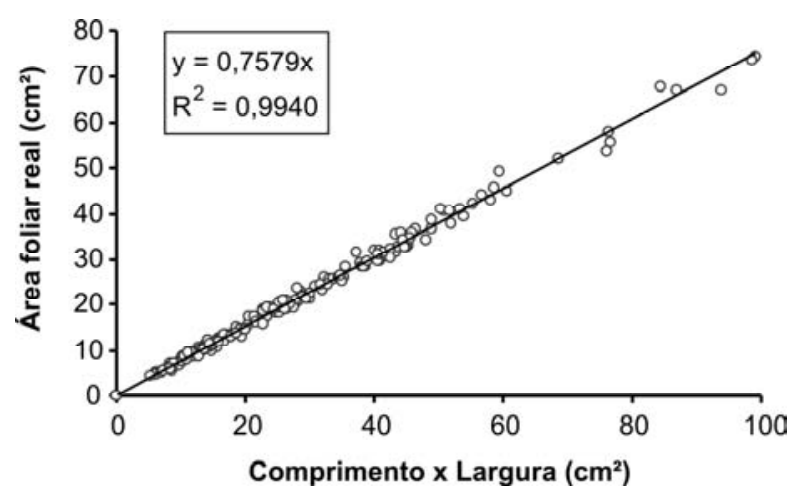

Figura 1 - Correlação entre a área foliar real e a estimativa produzida com o produto do comprimento ao longo da nervura central pela largura máxima das folhas de $A$. deflexus - casa de vegetação + campo. Piracicaba, 2005.

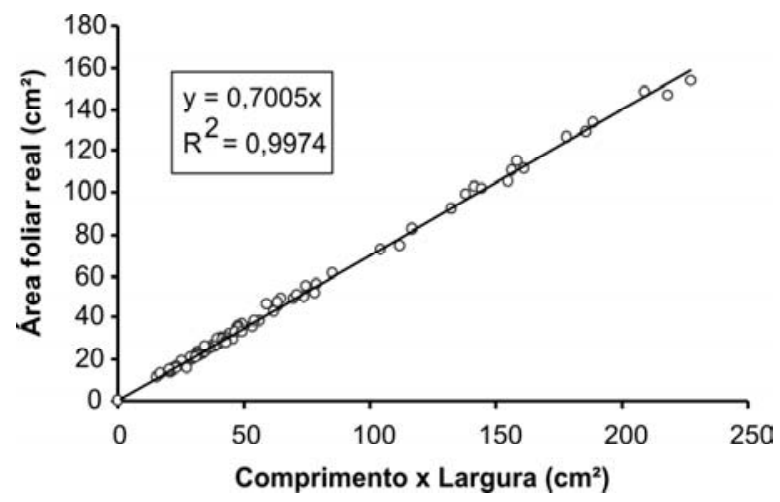

Figura 3 - Correlação entre a área foliar real e a estimativa produzida com o produto do comprimento ao longo da nervura central pela largura máxima das folhas de $A$. retroflexus - casa de vegetação. Piracicaba, 2005.

Nesse sentido, Aguilera et al. (2004) observaram que plantas de Siegesbeckia orientalis que cresceram sob condição de $50 \%$ de sombreamento apresentaram área foliar significativamente superior à das plantas que cresceram a pleno sol. Esses autores observaram rápido aumento da superficie fotossinteticamente ativa como forma de as plantas assegurarem o melhor aproveitamento das baixas intensidades luminosas. Ess es resultados também foram observados por Vlahos et al. (1991) e Farias et al. (1997).

Em geral, os valores de ajuste da reta obtidos neste trabalho, para as plantas daninhas do gênero Amaranthus, foram inferiores aos obtidos por Bianco et al. (1983) para Wissadula subpeltata, de 0,8549; e Bianco et al. (2003) para Typha latifolia, de 0,9651; entretanto, eles

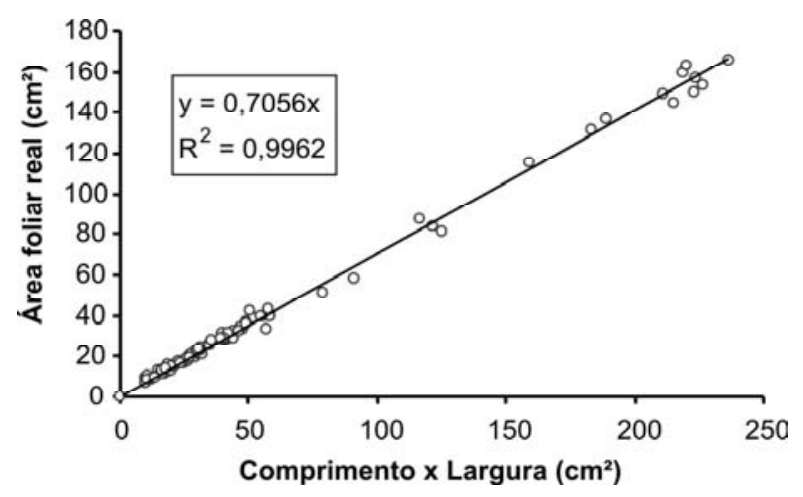

Figura 2 - Correlação entre a área foliar real e a estimativa produzida com o produto do comprimento ao longo da nervura central pela largura máxima das folhas de $A$. hybridus - casa de vegetação. Piracicaba, 2005.

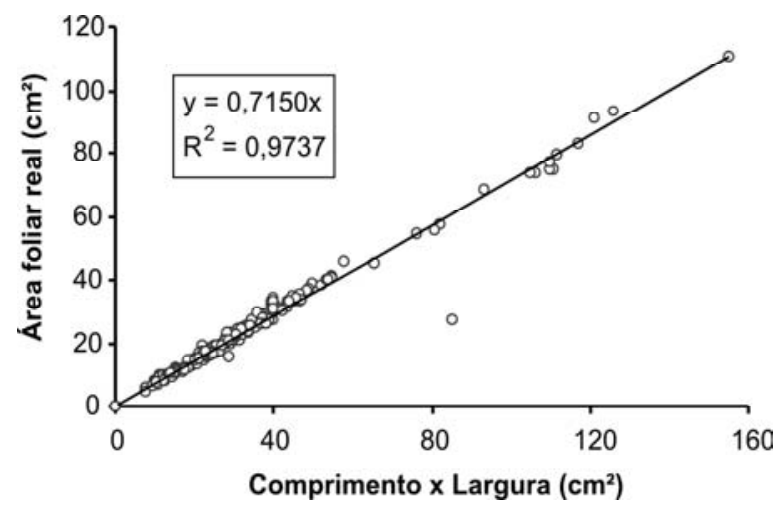

Figura 4 - Correlação entre a área foliar real e a estimativa produzida com o produto do comprimento ao longo da nervura central pela largura máxima das folhas de $A$. spinosus - casa de vegetação + campo. Piracicaba, 2005.

foram superiores ao obtido por Bianco et al. (2004) para Tridax procumbens, de 0,6008.

Com base nos resultados obtidos, há a possibilidade de condução de novos estudos científicos com as plantas daninhas do gênero Amaranthus sem a necessidade do uso de amostragem destrutiva, ou seja, tem-se a possibilidade de avaliar o desenvolvimento foliar ou a resposta fisiológica a agentes externos de um mesmo indivíduo, em todas as avaliações. Levando-se em conta o elevado ajuste estatístico nos dados, esse método pode ser usado para estimar a área foliar de plantas de Amaranthus, inclusive em campo.

Após a realização deste trabalho, concluiuse que: há espécies de plantas daninhas do gênero Amaranthus com tamanhos 


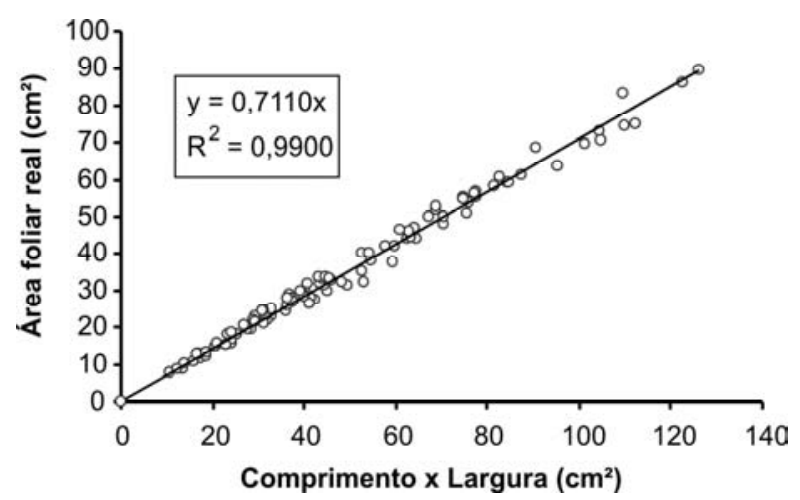

Figura 5 - Correlação entre a área foliar real e a estimativa produzida com o produto do comprimento ao longo da nervura central pela largura máxima das folhas de $A$. viridis - campo. Piracicaba, 2005.

diferenciais de folhas, sendo $A$. deflexus aquela que, em média, possui as menores dimensões; a equação linear passando pela origem $(A r=a .(C . L))$ foi adequada para ajustar a relação entre as medidas lineares do limbo e a área foliar real; e plantas que cresceram em casa de vegetação e em campo podem apresentar diferenças quanto ao tamanho de folhas ou no parâmetro $a$ da equação de ajuste da reta.

\section{AGRADECIMENTOS}

Os autores agradecem à Fundação de Amparo à Pesquisa do Estado de São Paulo (FAPESP), pela bolsa de estudos de mestrado concedida ao primeiro autor.

\section{LITERATURA CITADA}

AGUILERA, D. B.; FERREIRA, F. A.; CECON, P. R. Crescimento de Siegesbeckia orientalis sob diferentes condições de luminosidade. Planta Daninha, v. 22, n. 1, p. 43-51, 2004.

BIANCO, S.; PITELLI, R. A.; CARVALHO, L. B.

Estimativa da área foliar de Tridax procumbens usando dimensões lineares do limbo foliar. Planta Daninha, v. 22, n. 2, p.247-250, 2004.

BIANCO, S.; PITELLI, R.A.; PERECIN, D. Métodos para estimativa da área foliar de plantas daninhas. II. Wissadula subpeltata (Kuntze) Fries. Planta Daninha, v. 6, n. 1, p. 21-24, 1983.

BIANCO, S.; PITELLI, R. A.; PITELLI, A. M. C. M.

Estimativa da área foliar de Typha latifolia usando dimensões lineares do limbo foliar. Planta Daninha, v. 21, n. 2, p. 257261, 2003.

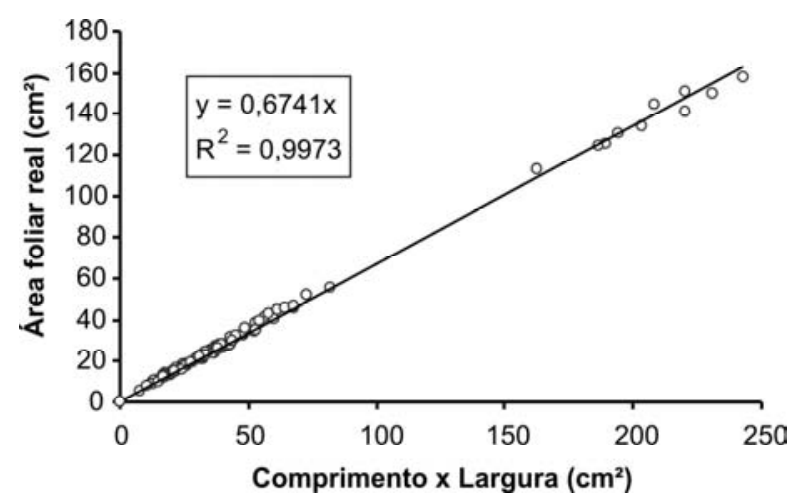

Figura 6 - Correlação entre a área foliar real e a estimativa produzida com o produto do comprimento ao longo da nervura central pela largura máxima das folhas de $A$. viridis - casa de vegetação. Piracicaba, 2005.

BIANCO, S. et al. Estimativa da área foliar de plantas daninhas. XIII - Amaranthus retroflexus L. Ecossistema, v. 20, n. 1, p. 5-9, 1995.

FARIAS, V. C. C. et al. Análise de crescimento de mudas de cedrorana (Cedrelina catenaeformis (Ducke) Ducke) cultivadas em condições de viveiro. R. Bras. Sementes, v. 19 , n. 2, p. 192-199, 1997.

FERNÁNDEZ, O. A. Manejo integrado de malezas. Planta Daninha, v. 5, n. 2, p. 69-75, 1982.

GUO, P.; AL-KHATIB, K. Temperature effects on germination and growth of redroot pigweed (Amaranthus retroflexus), Palmer amaranth (A. palmerii), and common waterhemp (A. rudis). Weed Sci., v. 51, n. 6, p. 869-875, 2003.

HESS, M. et al. Use of the extended BBCH escale - general for descriptions of the growth stages of mono-and dicotyledonous weed species. Weed Res., v. 37, n. 6, p. 433-441, 1997.

KISSMANN, K.G.; GROTH, D. Plantas infestantes e nocivas. 2. ed. São Paulo: BASF, 1999. v. 2. 978 p.

KLINGAMAN, T. E.; OLIVER, L. R. Palmer amaranth (Amaranthus palmerii) interference in soybean (Glycine max ). Weed Sci., v. 42, n. 4, p. 523-527, 1994.

KNEZEVIC, S. Z.; HORAK, M. J.; VANDERLIP, R. L. Relative time of redroot pigweed (Amaranthus retroflexus) emergence is critical in pigweed-sorghum [Sorghum bicolor (L.) Moench] competition. Weed Sci., v. 45, n. 4, p. 502508, 1997.

LUCCHESI, A. A. Utilização prática de análise de crescimento vegetal. An. ESALQ, v. 41, n. 1, p. 181-202, 1984. 
MARQUES, M. A. Potencial alelopático de resíduos de caruru (Amaranthus viridis) incorporado em três tipos de solo, sobre a germinação e crescimento inicial do algodoeiro (Gossypium hirsutum). 1992. 125 f. Dissertação (Mestrado em Solos e Nutrição de Plantas) - Universidade Federal de Lavras, Lavras, 1992.

MENGES, R. M. Allelopathic effects of Palmer amaranth (Amaranthus palmerii) on seedling growth. Weed Sci., v. 36, n. 3 , p. $325-328,1988$

MURPHY, S. D. et al. Effect on planting patterns and interrow cultivation on competition between corn (Zea mays) and late emerging weeds. Weed Sci., v. 44, n. 4, p. 865-870, 1996.

PERESSIN, V. A.; PITELLI, R. A.; PERECIN, D. Métodos para estimativa da área foliar de plantas daninhas. 4. Cassia tora L. Planta Daninha, v. 7, n. 2, p. 48-52, 1984.
RADOSEVICH, S.; HOLT, J. S.; GHERSA, C. Weed ecology: implications for vegetation management. New York: John Willey \& Sons, 1997. 589 p.

SPEHAR, C. R. Diferenças morfológicas entre Amaranthus cruentus, cv. BRS Alegria, e as plantas daninhas A. hybridus, A. retroflexus, $A$. viridis e $A$. spinosus. Planta Daninha, v. 21, n. 3, p. 481-485, 2003.

SPEHAR, C. R. et al. Amaranto BRS Alegria: alternativa para diversificar os sistemas de produção. Pesq. Agropec. Bras., v. 38, n. 5, p. 659-663, 2003.

VLAHOS, J. C.; HEUVELINK, E.; MARTAKIS, G. F. P. A growth analysis study of three Achimenes cultivars grown under three light regimes. Sci. Hortic., v. 46, n. 3-4, p. 275 $282,1991$. 